\title{
Optimum Technology Insertion into Systems Based on the Assessment of Viability
}

\author{
Peter A. Sandborn, Sr. Member IEEE, Thomas Herald, James Houston, and Pameet Singh ${ }^{*}$
}

\begin{abstract}
Product sustainment means keeping an existing system operational and maintaining the ability to continue to manufacture and field versions of the system that satisfy the original requirements. Sustainment also includes manufacturing and fielding revised versions of the system that satisfy evolving requirements, which often requires the replacement of technologies used to construct the original system with newer technologies. Technology insertion involves determining which technologies to replace during a design refresh, i.e., deciding the design refresh content, and deciding when that design refresh should take place. Technology replacement decisions are driven by a broad range of issues including performance, reliability, environmental impact, cost, and logistics, and when, or if other design refreshes will take place.

Traditional "value" metrics go part of the way toward providing a coupled view of performance, reliability and cost, but are generally ignorant of how product sustainment may be impacted. A metric that measures both the value of the technology refreshment and insertion, and its ability to support both the system's current and future affordability and capability needs including hardware, software, information and intellectual property is required.

This paper discusses a concept called viability. Viability is a measure of the producibility, supportability, and evolvability of a system and can serve as a metric for assessing technology insertion opportunities.
\end{abstract}

Index Terms - Viability, technology insertion, obsolescence, sustainment, Bayesian Decision Network.

\section{INTRODUCTION}

Each technology used in the implementation of a system (i.e., hardware, software, the technologies used to manufacture and support the system, information, and intellectual property) can be characterized by a life cycle that begins with introduction and maturing of the technology, and ends in some type of unavailability (obsolescence). Product sectors that do not control the supply chains for critical portions of their technological content, do not have the ability to influence the life cycle characteristics of these technologies, and therefore are forced to evolve as technology evolves. These product sectors include manufacturers of low-volume systems that are either manufactured for long periods of time and/or must be sustained for long periods of time. One of the critical technologies that these product sectors often cannot control are electronic components. Manufacturers in these product sectors must determine when to get off one technology's life cycle curve and onto another's in order to continue supporting existing systems and accommodate evolving system requirements, (Fig. 1).

Developing strategies and roadmaps ${ }^{1}$ that leverage technology evolution has been of interest for some time. The difficulty with historic roadmapping-based strategies is that they are: 1) inherently not application-specific, 2) tend to focus more on accurately forecasting the start of the technology life (when the technology becomes available and mature) and ignore the end of the technology life (obsolescence), and 3) the focus is often only on a 5 to 10 year predictive view, which does not provide enough insight to prepare full Total Ownership Cost views for 30, 40 and even 50-year programs. While conventional roadmapping approaches may be acceptable for those product sectors where there is no requirement for long-term sustainment (e.g., consumer electronics), it is not acceptable to sustainment-dominated product sectors. To address the application-specific impact of technology decisions on the life cycle of a system, the concept of viability is introduced. The objective of evaluating viability is to enable a holistic view of

\footnotetext{
* P.A. Sandborn and P. Singh are with the CALCE Electronic Products and Systems Center at the University of Maryland, College Park, MD 20742 USA.

T. Herald is with Lockheed Martin, Maritime Systems \& Sensors, Manassas, VA 20110 USA.

J. Houston is with Lockheed Martin Aeronautics Company, PO Box 748, Fort Worth, TX 76101 USA.

${ }^{1}$ By roadmapping, we mean both forecasting technology availability and unavailability (obsolescence).
} 

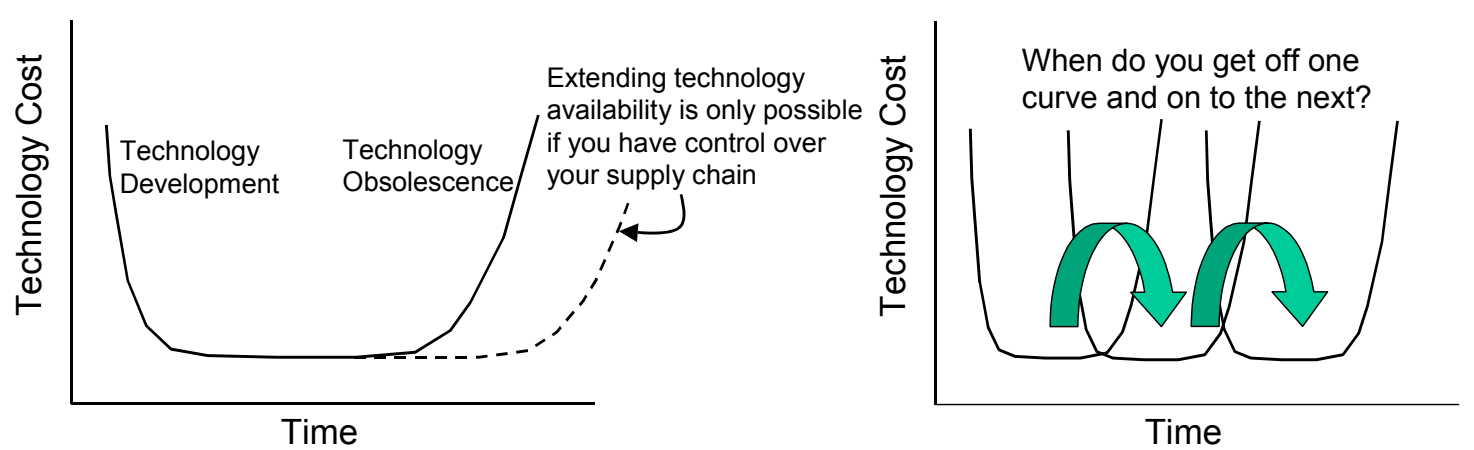

Fig. 1. Supporting systems and evolving requirements.

how the technology (and specific product) decisions made early in the design process impact the life cycle affordability of a system solution.

We define viability as a monetary and non-monetary quantification of application-specific risks and benefits in a design/support environment that is highly uncertain. ${ }^{2}$ Traditional "value" metrics go part of the way toward defining viability by providing a coupled view of performance, reliability and acquisition cost, but are generally ignorant of how product sustainment may be impacted. We require a viability metric that measures both the value of the technology refreshment and insertion, ${ }^{3}$ and the degree to which the proposed change impacts the system's current and future affordability and capability needs. As indicated above, this viability assessment must include hardware, software, information and intellectual property aspects of the product design. Viability therefore goes beyond just an assessment of the immediate, or nearterm impacts of a technology insertion, in that it evaluates the candidate design (or candidate architecture) over it's entire lifetime. ${ }^{4}$

Although viability can be defined in many ways, its underlying premise is that economic well-being is inextricably linked to the sustainability and of the system. According to studies conducted for the Air Force Engineering Directorate, [3]. Viability Assessment must include:

- Producibility - Ability to produce the system in the future based upon the "current" architecture and design implementation. (production and initial spares, not replenishment spares).

- Supportability - Ability to sustain the system and meet the required operational capability rates. This includes repair and re-supply as well as non-recurring redesign for supportability of the "as is" design implementation and performance.

- Evolvability (Future Requirements Growth) - Ability of the system to support projected capability requirements with the "current" design and avionics architecture. This includes capability implemented by hardware and software updates.

In June 2002, the US Air Force Aeronautical System Center (ASC) released the first RFP (Request For Proposal) specifically incorporating the concept of viability...[4].

\footnotetext{
${ }^{2}$ The definition of viability used in this discussion is a combination of economics and technical "value", but assumes that technical feasibility has already been achieved.

3 Technology refresh is used as a reference to system changes that "Have To Be Done" in order for the system functionality to remain useable. Technology insertion is a term used to identity the "Want To Be Done" system changes, which include both the new technologies to accommodate system functional growth and new technologies to replace and improve the existing functionality of the system, see [1].

${ }^{4}$ Note, viability has also been defined in the context of sustainable product realization as the combination of "function, economics, energy and environmental issues related to a product and its use" [2]. This definition is not inconsistent with the usage of the term viability in this paper, however, we have not specifically addressed environmental or energy issues herein, rather taking a more logistical view of sustainment, although, the general concepts presented herein for organizing the treatment of viability may be applicable to additional issues as well.
} 
3.2.2 Subfactor 2: Affordable Growth: 1. Redesign and/or procurement of changes. - What is your strategy to provide affordable changes (driven by component obsolescence, technology changes, and capability growth) over the life of the program?

This RFP may be a model for future solicitations from the defense community and represents a growing necessity for system designers and manufactures to pro-actively assess and plan for design refresh coupled with technology insertion.

The critical steps to making use of viability concepts in decision making are:

1) Identifying practical and measurable indicators of viability

2) Understanding how the indicators can be measured as a function of decisions made (and time passed)

3) Managing the necessary qualitative and quantitative information (with associated uncertainty) needed to evaluate the indicators

4) Performing the evaluation (possibly linked to other analyses/tools that are used early in the design process).

\section{EVALUATING VIABILITY}

The viability of each technology decision made, whether during the initial design of a product or during a redesign activity, should be evaluated. Viability is formulated from a mix of many things including the following two critical elements:

- Technology life cycle - the life cycle of various technology components (particularly electronic parts) has been modeled and can be represented, e.g., technical life cycle maturity, lifecodes [5], and obsolescence dates [6]. The life cycle forecast may be dynamic and change (with time) in response to some form of technology surveillance program. In general, this metric is not application specific (and only hardware-part specific at this time). This concept, however, could be extended in two ways: 1) for a "technology group", i.e., Computers, Memory, Bus Architectures, Sensors, Databases, Middleware, Operating Systems, etc. A "scaled-up" version of life cycle forecasting could provide a maturity metric for a technology grouping versus a specific application that uses one (or a combination of) technology groups; and 2) for non-hardware (nonelectronic) components such as software, information and intellectual property. ${ }^{5}$ No present methodologies or tools are capable of assessing a particular technology category and mapping evolutions against the 30,40 or 50 year life cycles that military systems and platforms are expected to perform.

- Associativity - the second element is the impact of a particular technology's modification on the specific application. As an example, one technology may be late in its life cycle, but the impact of changing it on the application is low (making it a candidate for consideration), i.e., it is not in the critical path for qualification or certification, it does not precipitate any other changes to the application, or it is modularized in such a way as to isolate its impact on the rest of the system, i.e., a timing module that provides synchronization can be easily changed without impacting on any other part in the system and thus no associativity. On the other hand, other technologies (at the same point in their life cycle) may be central to everything (such as an Operating System or Bus Architecture) and therefore have high associativity. ${ }^{6}$

\footnotetext{
${ }^{5}$ Electronic part obsolescence forecasting benefits from the commonality of parts in many systems, non-electronic part obsolescence can not take advantage of this situation, and therefore, common commercial approaches that depend on subjective supply chain information will likely be less useful for general non-electronic and non-hardware obsolescence forecasting [7]. A better approach for non-electronic component obsolescence may be life cycle curve fitting based approaches like, [6].

${ }^{6}$ This is important as we begin to consider the Affordability of a Technology Refresh or Insertion. It is important to identify the "Critical System Elements"; one way to do this is by using acquisition cost multiplied by the quantity needed in a system. For example, an operating system is relatively inexpensive and yet can be very critical. The 'value' of the operating system is NOT just its acquisition cost multiplied by its quantity, but should also sum all of the acquisition costs (multiplied by the respective quantities) of all effected parts of the system refreshment. This would be
} 
When formulating the indicators of viability, the methodology must accommodate the fact that there are many stakeholders who all possess different portions of the knowledge necessary to accurately evaluate the viability of a specific choice or decision. Another difficulty is that the information necessary to make the decision is generally incomplete, and consists of qualitative and quantitative content and their associated uncertainties. Thus viability evaluation represents an information fusion problem. ${ }^{7}$

\section{An EXAmPle of VAlue-Based Viability Decision Making Using BDNs}

Central to the viability information fusion problem is probabilistic reasoning. Most problems in the real world require that some simplifications be made to define their domain and make them amenable to solution. Many facts are left unknown or incompletely defined. Data without uncertainty for most problems is rarely possible to obtain and for the technology insertion problem at hand, uncertainties are a dominate consideration in the decision making process. The uncertainty could be inherent in the problem or it could represent a simplistic representation of its domain (or probably both). Usually there is some level of trade off between the acceptable data uncertainty and time or money spent to obtain the data. In general, there are three approaches to handle uncertainty [8]: 1) logicist, 2) neo-calculist, and 3) neo-probabilist. The logicist deals with non-numerical qualitative techniques (e.g., rule based). The neo-calculist rejects traditional probability calculus and creates new calculi (e.g., fuzzy logic). The neo-probabilist methods are normative, i.e., based on traditional probability calculus.

One way to accommodate the uncertainties and the mix of qualitative and quantitative inputs expected when assessing viability is to use a Bayesian Decision Network (BDN), [9]. Bayesian methods provide a formalism for reasoning about partial beliefs under conditions of uncertainty. In this formalism, propositions are given numerical parameters signifying the degree of belief accorded them under some body of knowledge, and the parameters are combined and manipulated according to the rules of classical probability theory (BDNs are a neo-probabilist approach to reasoning under uncertainty).

Bayesian Networks (also known as Bayes networks and causal probabilistic networks), provide a method to represent relationships between propositions or variables, even if the relationships involve uncertainty, unpredictability or imprecision. They may be learned automatically from data files, created by an expert, or developed by a combination of the two. They capture knowledge in a modular form that can be transported from one situation to another; it is a form people can understand, and which allows a clear visualization of the relationships involved.

Bayesian Decision Networks (BDNs) expands upon a Belief Network by allowing the visualization of a cause and effect relationship that relates decisions to endpoints rather than just parent to child nodes. By adding decision variables (things that can be controlled), and utility variables (things we want to optimize) to the relationships of a belief network, a decision network (also known as an influence diagram) is formed. This can be used to find optimal decisions, control systems, or make plans. This form of a network provides the means to take non-quantitative and semi-quantitative information and integrate it with quantitative information for decision making. Standard uncertainty analysis (e.g., Monte Carlo analysis) can only use quantitative information to make quantitative decisions. BDNs also have the ability to represent and respond to changing configurations (addition of evidence), i.e., "learn".

Traditional Bayesian Decision Networks emphasize conditional independence among random variables and only allow conditional probabilities to be defined for the various nodes. To assess viability, there may be a need to extend the decision networks into valuation networks that emphasize factorizations of joint probability distributions, [10]. Valuation networks are more general than BDNs and allow direct representation of all probability models.

done for each element in the system Bill of Materials, and thus a new priority sorting of the Bill of Materials would highlight the "System Critical Elements" by their cost impact to change. System Critical really refers to "Difficulty to Change based on Affordability". This is important for a second reason: this technology management represents a cost, and thus must focus on the system elements that drive the best affordability. Associativity helps clarify this issue.

${ }^{7}$ Information fusion is the seamless integration of information from disparate sources that results in an entity that has more value (and less uncertainty) than the individual sources of information used to create it. Fused information is information that has been integrated across multiple data collection "platforms" (soft and hard) and physical boundaries, then blended thematically, so that the differences in resolution and coverage, treatment of a theme, character and artifacts of data collection methods are eliminated. 
The traditional difficulty with BDNs is the computational burden associated with propagation (updating the probability associated with all the chance nodes in light of actual information or evidence). Recent advances in algorithms for solving NP-hard numerical problems have made the use of Bayesian propagation computations considerably more practical than they were only 10 years ago.

Examples of design decisions involving technology insertion include the use of a specific technology, the replacement of a part during a re-design, or performing or not performing a design refresh (i.e., Technology Refresh). In order to determine the viability of a design decision, we must determine how the design is changed at the event. The architecture of a BDN consists of a network of chance nodes connected by directed links (actually this is a Bayesian Belief Network - BBN). A chance node consists of a set of states (e.g., true or false; or low, medium or high) and the probability of those states (only the parents of a node and its children influence the node's probability table). BDNs are BBNs, extended with value (utility) nodes and decision nodes. A decision node accumulates the products of utilities and probabilities over the entire network and helps in making the decisions. A value node defines the value of a certain option being selected or a decision being made. Further, the nodes in the BDN can be organized into several categories that translate into the various types of constraints associated with (or more accurately, defining) viability:

- Translation of requirements (customer directed value) - the relative importance of meeting and/or exceeding customer requirements. Examples include weighting the value of exceeding a reliability specification versus meeting the specification.

- Application impact - how does the technology impact the specific application? Impact areas could include functionality, performance, capacity, reliability, cost, availability, qualification/certification, size, weight, etc.

- Design options - things that can be changed (decisions that can be made).

- Life cycle environment - the state of the technology being considered. Includes the life cycle characteristics of the technology, and application-specific information on your accessibility to the technology. Examples include whether or not a suitable replacement for an obsolete part exists, whether the IP required for an ASIC can be licensed or not, or whether a system design change is necessary (thus include the non-recurring engineering costs for the change).

Figure 2 shows an example BDN created for exploring the replacement of parts at a re-design activity. In this example, the decision will govern whether the part is changed (replaced or upgraded) or not changed at a design refresh and will depend (not only on the obsolescence attributes of the specific part), but also on the "value" realized by the system in changing the part (economic, performance, and reliability). The simple example shown enables sharing and understanding among many heterogeneous stakeholders (procurement, design, manufacturing, etc.) using both qualitative and explicit data. The BDN allows these stakeholder's views to be captured in the decision process. In addition, an advantage enabled by BDNs is the ability to propagate decisions in multiple directions, i.e., besides determining the highest value action to take with regard to a particular part at a re-design, it could also enable fixing a specific action on a specific part (a very realistic situation) and solving for the best actions for the other parts, or even the best refresh date.

This concept can then be applied to any hierarchical level of the system under consideration. For example, actions must be taken on an aircraft avionics suite to maintain viability, however, the manufacturer of the radar must also maintain viability. The manufacturer of the controls for the radar must also remain viable, until the lowest element is being considered. Thus, applicability is multi-tiered.

A BDN based approach could easily be made to complement existing tradeoff and planning tools, e.g., [11], [12]; and CAIV (Cost As an Independent Variable) methodologies [13]. Note, BDN approaches are also applicable to the technology forecasting part of the problem. Meixell and $\mathrm{Wu}$ [14] use BDNs in leading indicator models for predicting demand (and thus obsolescence) in semiconductor markets using a Bayesian likelihood function based on observed early demand to update probabilities of the parameters associated with a demand model.

\section{BROAD APPLICABILITY IN THE COMMERCIAL SECTOR AND BEYOND}

Besides the obvious applicability of viability-based technology insertion optimization for military and commercial avionics (and other defense related electronic systems), the value of such a capability could be readily extended to non-electronic systems. For example, the world is full of computer networks that are 

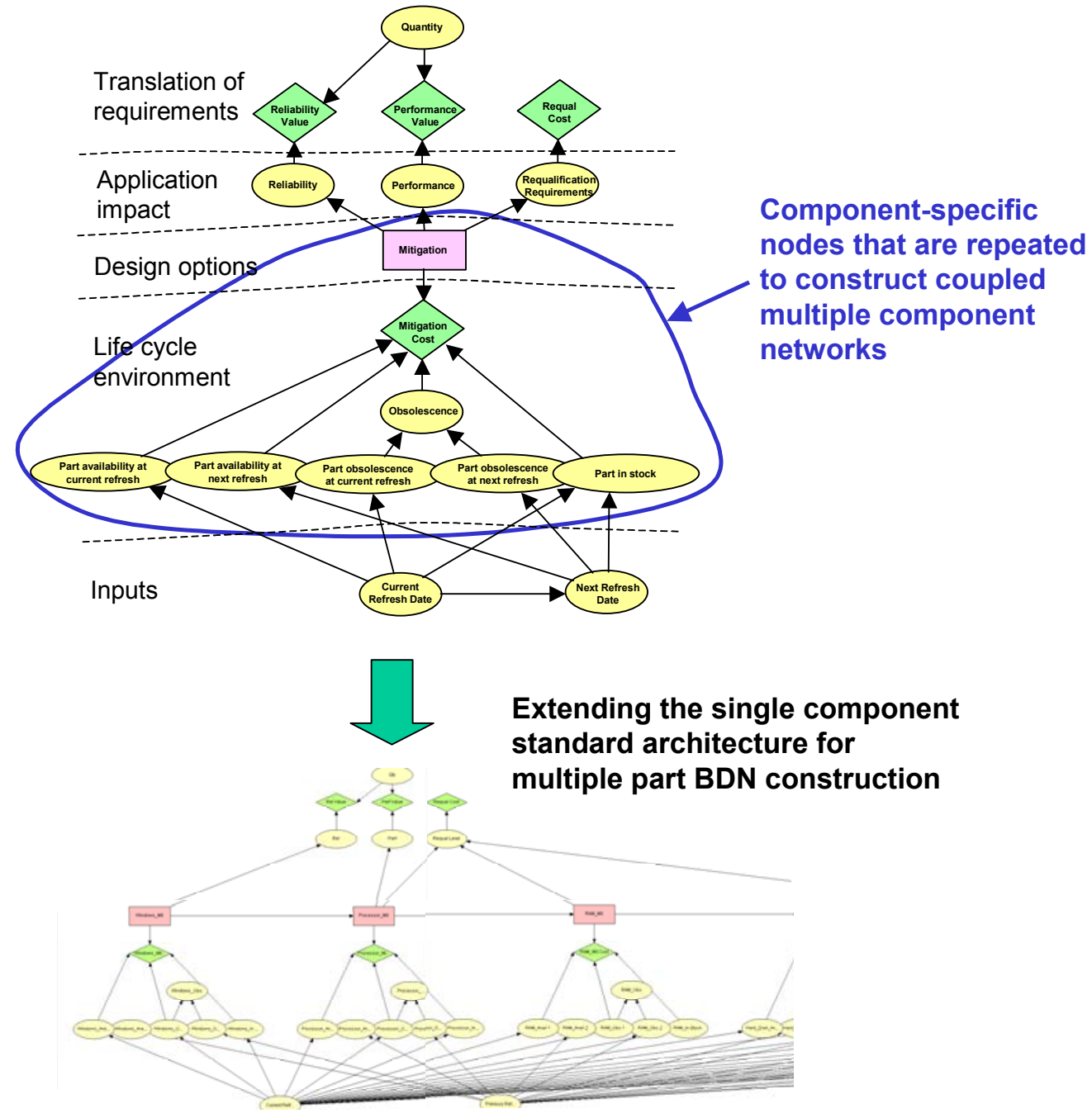

Fig. 2. Example Bayesian Decision Network (BDN) for determining whether to replace or not replace electronic components at a specific re-design point (left). The single component is included within a multicomponent net (right).

continuously becoming obsolete (i.e., portions are probably obsolete before their first use, and portions are becoming obsolete continuously thereafter). Examples of these systems include networks for: air traffic control; telephone switching, and power grid management. The obsolescence of these systems means that 1) they become more "difficult" to sustain, and 2) they slide further from the evolving performance and/or functionality requirements placed on them. Exacerbating the problem, the computer network example represents a very interdisciplinary system consisting of hardware, software, information and intellectual property. So, when and how do you redesign and technology upgrade a system like a computer network? Can optimum redesign/technology insertion points be forecasted? If they could be forecasted, budgets could be adjusted to fit the forecasts and substantial life cycle cost savings might be possible.

\section{THE PAYOFF}

The payoff of being able to assess system viability is in enabling proactive life cycle planning that provides a program manager with the ability to predict as early as possible (while the input data is uncertain) how to best plan system sustainment. An ability to forecast optimal design refresh and technology insertion strategies that enables significant cost avoidance:

- more accurate allocation of budget earlier in program development phases

- more accurate guidelines for how systems are modified at design refreshes 
- improved operational availability

- more optimal problem mitigation approach decisions

- enables the opportunity for shared solutions across multiple systems and applications

- enables better supply chain management of sparing, since spares are only needed for a "specified time" before a replacement is identified. In fact, often this time is less than the typical reliability of the particular part, and thus it is replaced (for impending obsolescence) before failure as the new product is installed. Fewer spares are needed.

\section{REFERENCES}

[1] T. E. Herald, "Technology refreshment strategy and plan for application in military systems - A "howto systems development process" and linkage with CAIV," Proc. National Aerospace and Electronics Conference (NAECON), pp. 729-736, October 2000.

[2] D. Durham, "Predictive product realization: Bridging design and manufacturing through modeling and simulation," presentation to the National Defense Industrial Association System Engineering Modeling \& Simulation Committee, April 2003.

[3] B. Ardis, "Viable/affordable combat avionics (VCA) implementation update," Dayton Aerospace, Inc., June 2001.

[4] MD-CDL - Multi-Platform Common Data Link (http://www.pixs.wpafb.af.mil/asc.asp), 2002.

[5] i2 Technologies, Inc., http://www.tactech.com, 2002.

[6] R. Solomon, P. Sandborn and M. Pecht, "Electronic part life cycle concepts and obsolescence forecasting," IEEE Trans. on Components and Packaging Technologies, vol. 23, no. 3, pp. 707-713, December 2000.

[7] M. A. Howard, "Component obsolescence - It's not just for electronics anymore," Proc. FAA/DoD/NASA Aging Aircraft Conference, San Francisco, CA, September 2002.

[8] J. Pearl, Probabilistic Reasoning in Intelligent Systems: Networks of Plausible Inference, Morgan Kaufmann Publishers, San Mateo, CA, 1988.

[9] F. V. Jensen, Bayesian Networks and Decision Graphs, Springer Verlag, New York, NY, 2001.

[10] P. P. Shenoy, "Valuation-based systems for Bayesian decision analysis," Operations Research, vol. 40, no. 3, pp. 463-484, May-June 1992.

[11]P. Sandborn and P. Singh, "Electronic part obsolescence driven design refresh optimization," Proc. FAA/DoD/NASA Aging Aircraft Conference, San Francisco, CA, September 2002.

[12] T. Herald, "Technology refreshment of COTS-intensive system architectures," INCOSE Meeting, WMA Chapter, January 14, 2003. R2T2 - Rapid Response \& Technology Trade Study Tool, Lockheed Martin Corporation.

[13] J. G. Land, "Differences in philosophy - Design to cost vs. cost as an independent variable," Program Manager Magazine, pp. 24-28, March-April 1997.

[14]M. Meixell and S.D. Wu, "Scenario analysis of demand in a technology market using leading indicators," IEEE Transactions on Semiconductor Manufacturing, vol. 14, no. 1, pp 65-78, 2001. 